1: Final Report DE-FG02-07ER46361 The University of Oklahoma

\title{
2: Ro-vibrational Relaxation Dynamics of PbF Molecules
}

Neil Shafer-Ray (shaferry@physics.ou.edu)

3: April 8, 2011 (Covering budget period 1/1/2008 - 1/31/2011, \$433,959

4: Participating Laboratory: Brookhaven National Laboratory (Gregory Hall and Trevor

Sears)

\section{5) GOALS AND OBJECTIVES}

In 1950 Purcell and Ramsey[1] suggested that the electron might have a CP-violating electric dipole moment (e-EDM) proportional to its spin angular momentum. This possibility initiated an ongoing hunt for the e-EDM that has been spurred on by the recognition of the importance of CP-violation to the formation of a matter-dominated universe[2] as well as a difference in magnitude of the Supersymmetric[3] and Standard Model[4] prediction for its value. The current limit on the e-EDM is $1.6 \times 10^{-27} \mathrm{e} \cdot \mathrm{cm}$ as determined in a Ramsey beam resonance study of the $\mathrm{Tl}$ atom[5].

The PbF molecule provides a unique opportunity to measure the e-EDM. The molecule's odd electron, heavy mass, and large internal field combine to give it an intrinsic sensitivity to an e-EDM that is over three orders of magnitude bigger than that of the $\mathrm{Tl}$ atom[6]. In addition to this increased intrinsic sensitivity, the ground state of the $\mathrm{PbF}$ molecule allows for a "magic" electric field at which the magnetic moment vanishes[7]. All of these advantages create an opportunity to significantly lower the current limit on the e-EDM. These advantages can only be realized if an intense source of ground-state PbF molecules can be created and detected with high efficiency. The scope of this project is to (1) create a rotationally cold molecular beam source of $\mathrm{PbF},(2)$ achieve a continuous ionization scheme for sensitive state selective detection of the $\mathrm{PbF}$ molecule.

\section{6) ACCOMPLISHMENTS}

This research effort has been supported by the DOE since January of 2007. It has supported two years of Ph.D. work by graduate student Milinda Rupasinghe and four years of Ph.D. research support for graduate student Christopher McRaven. In addition, three publications. In addition, it resulted in four publications. The details of results posted in each of these publications are given below:

2.1) The Effect of the Geometric Phase on the Possible Measurement of the Electron's Electric Dipole Moment Using Molecules Confined by a Stark-Graviational Trap,Milinda Rupasinghe, N.E. Shafer-Ray, Phys. Rev. A, 78033427 (2008)

It is possible to envision a molecular $e-E D M$ experiment which probes $P b F$ molecules confined to a region in space by a non-uniform electric field, as illustrated in Fig 2.1. In such an experiment, one would polarize the trapped molecules creating a coherent superposition of 
states differing only in the sign of the projection of total angular momentum onto an electric field axis. If the electron were to have an electric dipole moment (i.e., if an $e-E D M$ exists) then these two states would have different energies and evolve creating a measurable time-dependent quantum beat.

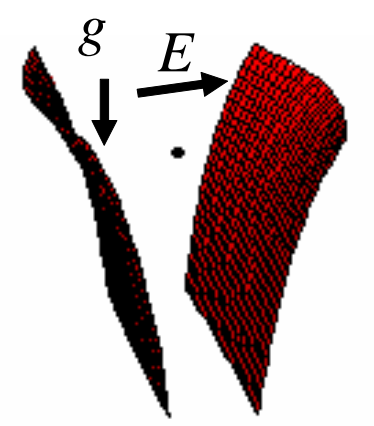

FIG 2.1: Electrodes that could be used to create a trap which confine $\mathrm{PbF}$ molecules in a potential created by the Earth's gravitational field and an anisotropic electric field.

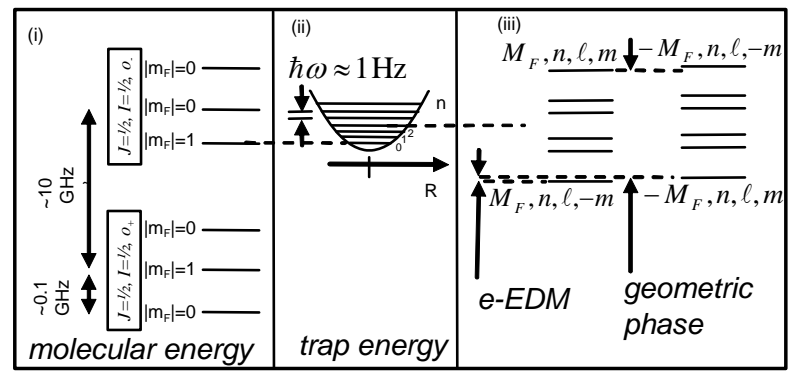

FIG 2.2: Energy level structure of ${ }^{208} \mathrm{~Pb}^{19} \mathrm{~F}$ molecules confined to the Stark-gravitation trap of FIG 2.1.
The original aim of our previously funded DOE research was to investigate the rotational-relaxation dynamics of a hot source of $\mathrm{PbF}$ in a cold bath of He. If these dynamics were to be favorable, it would allow us to produce a sub-Kelvin source of molecules that could be loaded into a trap. We soon realized, however, that to perform an $e-$ $E D M$ experiment would require more than just a trapped population of PbF. Specifically, the $e-E D M$ measurement would be limited by a coherence time $\tau$ that is much shorter than the lifetime of molecules in the trap. Instead $\tau$ would be determined a geometric phase effect that couples the angular momentum of the center-of-mass motion of a molecule to its internal angular momentum.

In this work we used the formalism of Longuett-Higgens[8] to derive an effective Hamiltonian for this effect and determined the coherence time $\tau$. Specifically we find that geometric phase couples the center-ofmass motion of a trapped molecule to its internal angular momentum via the effective Hamiltonian

$$
\Omega_{g e o}(\vec{r})=\frac{2 m \hbar i}{M} \sum_{n=1}^{3}\left[\left(\left(\nabla_{n} \frac{\vec{e}}{e}\right) \times \frac{\vec{e}}{e}\right) \cdot \hat{z}\right] \nabla_{n} .
$$

Here $m$ is the projection of the molecule's internal angular momentum on the electric field axis, $M$ is the mass of the molecule, $\vec{r}$ is the position of the molecule in the trap, $\nabla_{n} \in$ $\left\{\partial_{x}, \partial_{y}, \partial_{z}\right\}$ and $\vec{e}=\hat{z}+\hat{E}$ is the sum of the unit vectors giving the direction of the laboratory frame axis and direction of the electric field. Because the expectation value of $\Omega_{g e o}(\vec{r})$ over the center-of-mass wave functions of the trapped molecules is not zero, the $\pm m$ degeneracy expected by CP symmetry is lifted.

The expected energy level structure of a molecule trapped in a Stark gravitational trap is shown in Fig 2.2. Rotational and hyperfine energies are expected to be much greater than the trap energies which are typically of order $1 \mathrm{~Hz}$. The Geometric Phase effect further splits these energy levels. It is interesting to note that the trap still exhibits a degeneracy 
between states which differ only by the sign of the total (internal plus center-of-mass) angular momentum.

If one could create a cloud of molecules so cold that coherent superpositions of two centerof-mass states could be made, the geometric-phase induced-level structure could actually be useful, providing a well-understood energy level structure of comparable size to the $e-\mathrm{EDM}$ effect. However, such extraordinary control over the center-of-mass motion is currently limited to the alkali atoms For the experiment we visualize, the geometric phase effect simply limits the coherence time of an $e$-EDM experiment to $\tau \approx\left[\frac{T}{23 m K}\right]^{-2}$ sec. This is an unfortunate result because our proposed cooling techniques do not produce a population of $\mathrm{PbF}$ molecules in the $\mathrm{mK}$ temperature range. Thus even if successful in cooling and trapping $\mathrm{PbF}$ molecules, our coherence time would not exceed that of a molecular beam experiment. For this reason, a change in direction of the research project was requested and granted by the DOE. Specifically, our effort was shifted to the development of continuous resonance-enhanced multiphoton ionization detection scheme.

2.2) Characterization of the $X_{1}, A, B, D, E, E 1$, and F1, and F states of PbF. J Mol Spec, 262, 89 (2010.)

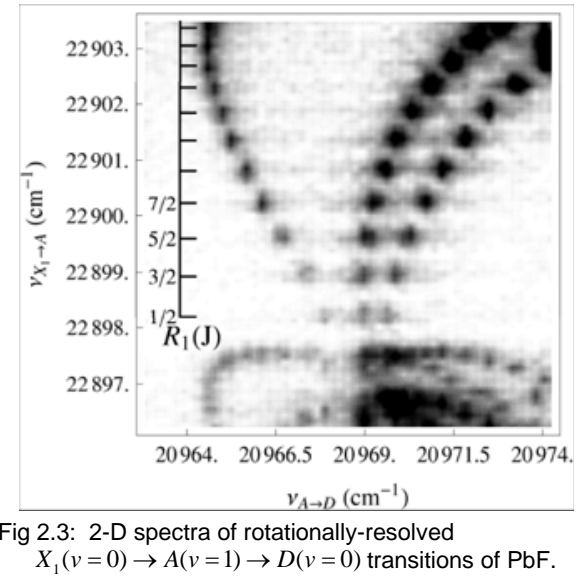

We have completed a detailed spectroscopic study of all known electronic states of the $\mathrm{PbF}$ molecule except the $C$ state (which we can not detect with rotational-state resolution.) These states were detected with 2-dimensional resonanceenhanced multiphoton ionization via the process $X_{1}$ ${ }^{2} \Pi_{1 / 2} \rightarrow A^{2} \Sigma_{1 / 2} \rightarrow[B(v=0), D(v=0), E(v=$ $0,1)$, or $F(v=0,1)] \rightarrow P b F^{+}$. Typical data spectrum are shown in Fig. 2.3. With this REMPI scheme, we have corrected values of $T_{e}$ by as much as $100 \mathrm{~cm}^{-1}$, we have determined heretofore unknown rotational constants, we have assigned term symbols to the $C$ and $D$, states, and determined the value of $\Omega$ for the $E$ and $F$ states. We have also confirmed the parity of the $B$ state. One interesting finding is that the assumed electronic mixing of the $X_{1}, X_{2}$, and $A$ states is not as simple as once thought. In a simple three-state ${ }^{2} \Pi_{1 / 2}-{ }^{2} \Pi_{3 / 2}-{ }^{2} \Sigma_{1 / 2}$ mixing scheme, the sum of the ratio of omega-doubling constant to rotational constant $p / B$ is \pm 2 (see $[6,9]$.) These ratios are given in the table below for the $X_{1}, A$, and $D$ states.

$$
\begin{array}{c|cccc} 
& X_{1}(v=0) & A(v=7) & A(v=0) & D(v=0) \\
\hline p / \beta & -0.605 & 2.60 & 2.996 & -1.04
\end{array}
$$

This table, as well as the energy levels of the electronic states suggest that the $X_{1}^{2} \Pi_{1 / 2}, X_{2}^{2} \Pi_{3 / 2} \rightarrow A^{2} \Sigma_{1 / 2}(v=7)$ system forms one strongly mixed ${ }^{2} \Pi-{ }^{2} \Sigma$ system whereas the $D^{2} \Pi_{1 / 2}, C^{2} \Pi_{3 / 2} \rightarrow A^{2} \Sigma_{1 / 2}(v=0)$ forms another. In light of this finding, a re-evaluation of the sensitivity of the molecule's sensitivity to parity violation is needed. 
2.3) Direct measurement of the lifetime of the D state of PbF, P. Sivakumar, C.P. McRaven, Dustin Combs, N.E. Shafer-Ray, and Victor Ezhov, Phys. Rev. A 77, 062508 (2008.)

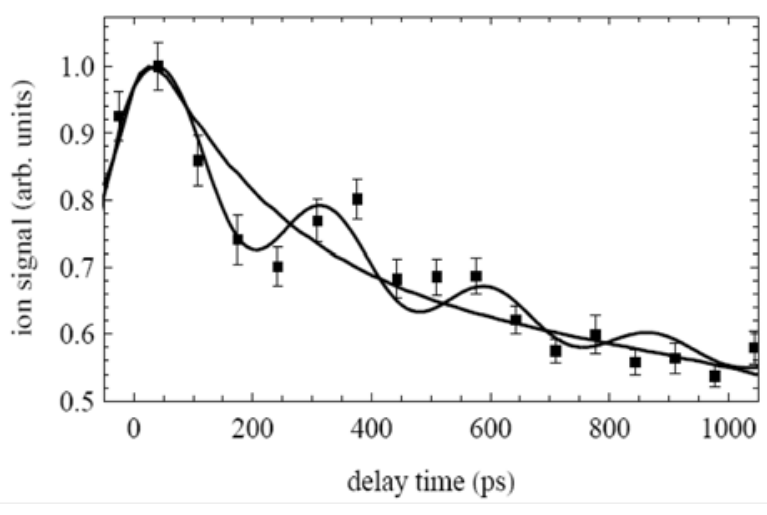

Fig 2.4: Time-dependent decay of the $\mathrm{D}$ state of $\mathrm{PbF}$.

The $A$ state is the only electronically excited state of $\mathrm{PbF}$ known to have a lifetime that is sufficiently long to allow for state-selective detection. Unfortunately, direct ionization of this state proves to be impractical: The uv laser radiation that is blue enough to ionize the $A$ state also directly ionizes the ground state via an efficient $1+1$ ionization process. This non-state-selective one-color process completely swamps any state-selective twocolor $1+1$ ionization process that might occur. This fact led us to the $1+1+1$

doubly resonance enhanced ionization scheme

$$
X \underset{436 \mathrm{~nm}}{\rightarrow} A \underset{476 \mathrm{~nm}}{\rightarrow} D \underset{532 \mathrm{~nm}}{\rightarrow} P b F^{+} .
$$

Knowledge of the $D$-state lifetime is critical to the design of an optimal laser system to implement this ionization scheme. Unfortunately the bandwidth of the Nd:YAG pumped dye laser system used to discover the $A \rightarrow D \rightarrow P b F^{+}$ionization pathway is not sufficiently narrow to make a conclusive statement about the $D$-state lifetime. For this reason we coaxed our 7-ns Nd:YAG laser system to perform a time domain measurement with picosecond resolution. To accomplish this we took advantage of the temporal mode structure of our unseeded Nd:YAG laser-pumped dye laser system. This structure is observed in an auto-correlation measurement that monitors the 251-nm radiation resulting from sumfrequency generation of 532-nm and 477-nm laser radiation in a BBO crystal as a function of an optical delay between the two input wavelengths. Determination of the lifetime of the $D$ state of $\mathrm{PbF}$ is made by observing the $\mathrm{PbF}^{+}$ionization signal as a function of the delay between the 477-nm and 532-nm laser radiation. The resulting time-dependent ionization signal (Figure 1) indicates a lifetime of $250 \pm 150 \mathrm{ps}$. The $D$-state lifetime is short enough to dramatically reduce the efficiency of an ionization scheme utilizing continuous wave radiation. For this reason, we employ a pseudo-continuous source of laser radiation (see section 2.4.)

2.4) Observation of continuous ionization of the PbF molecule, Mol Phys, 28, 927 (2011)

The combination of mass discrimination and near-unit quantum efficiency for detection makes Resonance Enhanced Multiphoton Ionization (REMPI) one of the most sensitive molecular detection techniques[? ]. However, although the technique has a very high peak sensitivity, it is typically carried out with nanosecond pulsed laser systems which operate at 
10 to $10,000 \mathrm{~Hz}$ repetition rate. These pulsed laser systems have two disadvantages. The first disadvantage is a very low effective duty cycle. This duty cycle is given by the ratio of time it takes for molecules to cross the laser radiation (typically microseconds) to the time between pulses of laser radiation (typically 0.1 to $100 \mathrm{~ms}$.) Thus a factor between 100 and $10^{4}$ in data collection rate is lost. A second disadvantage is that pulsed laser systems often have limited resolution given, in the best of circumstances, by the Fourier transform of the time-dependence of the radiation pulse.

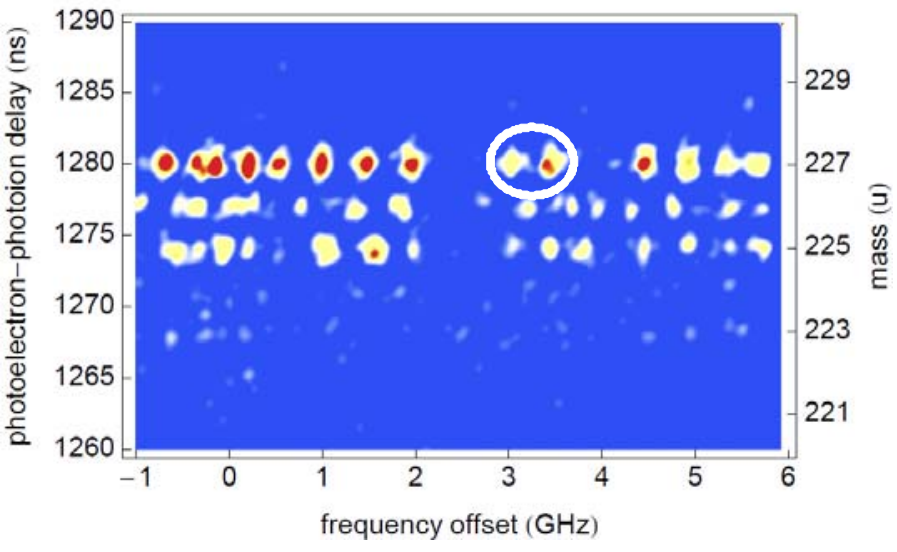

Figure 2.5: Hyperfine resolved pseudo continuous ionization spectra of The $X_{1} \rightarrow A$ transition of the three common isotopes of $\mathrm{PbF}$ figure 1

Fortunately a new generation of easy to use picosecond OPO systems have been developed (largely to aid in CARS spectroscopy.) These pseudo continuous systems produce broadly tunable picosecond pulses of laser radiation at a repetition rate of typically $50-100 \mathrm{MHz}$. This rate is sufficiently fast that, even for the case of a tightly focussed beam, molecules are exposed to several pulses of radiation before drifting out of the probe region. By copropagating this radiation with $\mathrm{cw}$ laser radiation, one can create stateselective ionization radiation that occurs with high probability each time a molecule enters the probe region. Here we have implemented such an ultra-sensitive cw-REMPI scheme to detect the PbF molecule.

Our ionization scheme begins with a blue diode laser (Toptica DL100) that produces $10 \mathrm{~mW}$ of radiation at $437 \mathrm{~nm}$. This radiation is used to excite the $X_{1}(v=0) \rightarrow A(v=1)$ transition. The second harmonic output of a $\mathrm{Nd}: \mathrm{YVO}_{4}$ laser (High-q laser picoTrain) is then used to pump an OPO system (APX Levante Emerald) to produce 1.4 watts of $863 \mathrm{~nm}$ laser radiation which arrives in 6 ps pulses at a repetition rate of $76 \mathrm{MHz}$. The $\mathrm{Nd}: \mathrm{YVO}_{4}$ harmonic separation optics have been modified to access the residual $1064 \mathrm{~nm}$ from the SHG crystal internal to the laser. This residual laser radiation (3.0 watts) is mixed in an type II SFG LBO crystal (Red Optronics) to produce $700 \mathrm{~mW}$ of $476 \mathrm{~nm}$ light.

The co-propagating $437 \mathrm{~nm}$ and $476 \mathrm{~nm}$ laser radiation is tightly focussed onto the molecular beam. The $476 \mathrm{~nm}$ laser radiation both excites the $A(v=1) \rightarrow D(v=0)$ transition and ionizes the $\mathrm{D}$ state. The $\mathrm{PbF}^{+}$ions are accelerated to a microchannel plate detector. Because the time-of flight of the PbF molecules $(1 \mu \mathrm{sec})$ is much longer than the $0.013 \mu \mathrm{sec}$ repetition period of the OPO, it is not practical to use the laser pulse as a trigger signal. Instead a symmetric electron detector has been built and is placed opposite to the ion detector. By detecting the electron in coincidence with the ion, mass resolution can be obtained. Both the electron and ion detectors are identical with roles that can be reversed by simply changing the bias voltages. Figure 2.5 shows a spectra that demonstrates the sensitivity of the technique. These spectra are taken from a rotational hot $\left(T_{\text {rot }}=1400 \mathrm{~K}\right)$ effusive beam. Nevertheless,individual hyperfine transitions are clearly resolved. The circled transitions 
are Q-branch transition from the ground $\mathrm{J}=1 / 2$ level of ${ }^{208} \mathrm{~Pb}^{19} \mathrm{~F}$. The cw-REMPI scheme we have implemented currently represents a roughly 50-fold improvement in data collection rate and a 10-fold improvement in the spectral resolution achieved by seeding our $10 \mathrm{~Hz}$ Nd:YAG pumped dye laser system with a diode laser.. Optimization is continuing and we are confident that this technology could be improved to create even more dramatic increases in sensitivity, both to $\mathrm{PbF}$ and to other systems. Even the 50-fold improvement in sensitivity we have already observed could have a profound impact on a number of applications that require the ultra-sensitive detection of atoms and molecules.

\section{7) PUBLICATIONS ACKNOWLEDGING THIS GRANT}

1. The Effect of the Geometric Phase on the Possible Measurement of the Electron's Electric Dipole Moment Using Molecules Confined by a Stark-Graviational Trap,Milinda Rupasinghe, N.E. Shafer-Ray, Phys. Rev. A, 78033427 (2008)

2. Direct measurement of the lifetime of the D state of PbF, P. Sivakumar, C.P. McRaven, Dustin Combs, N.E. Shafer-Ray, and Victor Ezhov, Phys. Rev. A ry, 062508 (2008.)

3. Characterization of the $X_{1}, A, B, D, E, E 1$, and F1, and F states of PbF. J Mol Spec, 262, 89 (2010.)

4. Observation of continuous ionization of the PbF molecule, Mol Phys, 28, 927 (2011)

\section{8) PERSONNEL SUPPORTED UNDER THIS GRANT}

The primary mover and shaker on this grant was Christopher McRaven, who's Ph.D. thesis included a large part of the development of pseudo-continuous resonance enhanced multi-photon ionization, the major accomplishment of this grant. Other undergraduate and graduate students floated into and out of the grant as tasks needed to be accomplished that required extra effort.

\begin{tabular}{|c|c|c|c|c|c|c|c|}
\hline & & \multicolumn{5}{|c|}{ months of support } \\
\hline status & name & 07 & 08 & 09 & 10 & 11 & total \\
\hline undergraduate & Leah Trafford & 2 & 1 & - & - & - & 3 \\
\hline undergraduate & Jacob Stinnet & - & - & 2 & - & - & 2 \\
\hline graduate student & Prayanka Rupasinghe & 1 & 4 & - & - & - & 5 \\
\hline graduate student & Sivakumar Poopalasingam & - & 4 & - & - & - & 4 \\
\hline graduate student & Christopher McRaven & - & 8 & 5 & 12 & 3 & 28 \\
\hline graduate student & Tao Yang & - & - & 1 & - & - & 1 \\
\hline graduate student & Haoquan Fan & - & - & - & - & 1 & 1 \\
\hline PI & Neil Shafer-Ray & 1 & 1 & 1 & - & - & 3 \\
\hline 1
\end{tabular}

${ }^{1}$ While supported by the DOE, these students worked on projects solely related to this grant. Funding was switched to other grants for portions of their work not related to the DOE project. 


\section{9) OTHER SUPPORT}

The researcher supported under this grant has led to a newly sensitive detection scheme with what could be profound implications for many applications requiring ultra-sensitive detection of gas-phase molecules. The research results are now being used for an e-EDM measurement supported by the NSF (NSF-PHY0602490.)

\section{10) COST STATUS}

See attached documentation. 
University of Oklahoma Norman Campus

Office of Research Services

Department Status Report

Fund Code

Department Number

50070

105053400

Actual Costs as of

Commitments as of

$3 / 31 / 2011$

$4 / 13 / 2011$

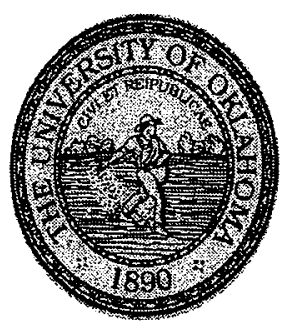

CLOSED

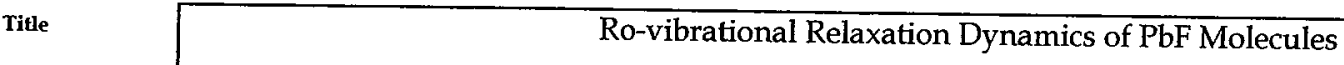

Principal Investigator

Department

College
Professor Neil Shafer-Ray

Physics \& Astronomy

Arts \& Sciences

$\begin{array}{ll}\text { Agency } & \text { US-DOE } \\ \text { Document No. } & \text { DE-FG02-07ER46361 } \\ \text { Federal Flow-Thru Funds } & \text { No } \\ \text { Type of Award } & \text { Cost Reimbursable } \\ \text { Budget Period } & 02 / 01 / 2009-01 / 31 / 2011 \\ \text { Project Period } & 02 / 01 / 2007-01 / 31 / 2011\end{array}$

Agency

02/01/2007 - 01/31/2011

\section{Budget}

Categories

Current

Operating

Budget

March-11

Cumulative

Costs

Commitments

Uncommitted

Modified Total Direct Cost (MTDC)

Salaries \& Wages

Fringe Benefits

Project Materials

Domestic Travel

Foreign Travel

Professional Services

Technical Meetings

Other Direct Costs

Subtotal: MTDC

\section{Non-MTDC}

Equipment

Fabricated Equipment

Tuition Rate

Subtotal: Non-MTDC

Total Direct Cost (TDC)

F\&A Costs @ 48\% MTDC

Total Costs
Report Prepared By

Telephone No.

Mailing Address
$\$ 104,237.14$

$\$ 11,883.02$

$\$ 43,850.32$

$\$ 21,781.49$

$\$ 5,271.03$

$\$ 0.00$

$\$ 0.00$

$\$ 6,520.80$

$\$ 193,543.80$
$\$ 0.00$

$\$ 0.00$

$\$ 0.00$

$\$ 0.00$

$\$ 0.00$

$\$ 0.00$

$\$ 0.00$

$\$ 0.00$

$\$ 0.00$

$\begin{array}{r}\$ 104,237.14 \\ \$ 11,883.02 \\ \$ 43,850.32 \\ \$ 21,781.49 \\ \$ 5,271.03 \\ \$ 0.00 \\ \$ 0.00 \\ \$ 6,520.80 \\ \hline \$ 193,543.80\end{array}$

$\$ 9,503.50$

$\$ 341,057.96$

$\$ 433,959.00$
$\$ 92,901.04$

$\$ 341,057.96$

$\$ 936.32$
$\$ 950350$
$\$ 121,390.73$

$\$ 16,619.93$

$\$ 147,514.16$

$\$ 92,901.04$

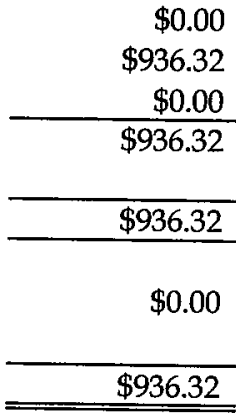

$\$ 433,959.00$

$\begin{array}{r}\$ 0.00 \\ \$ 0.00 \\ \$ 0.00 \\ \$ 0.00 \\ \$ 0.00 \\ \$ 0.00 \\ \$ 0.00 \\ \$ 0.00 \\ \hline \$ 0.00\end{array}$

$\$ 0.00$

$(\$ 0.00)$

$\$ 0.00$

$(\$ 0.00)$

$\$ 0.00$

$\$ 0.00$

$\$ 0.00$

$(\$ 0.00)$
$\$ 0.00$

$\$ 0.00$

$\$ 0.00$

$\$ 0.00$

$\$ 0.00$

$(\$ 0.00)$
Earnest (EJ) Johnson, CRA (405) 325-4448

Three Partners Place, Suite 150
Title

Fax No.

E-mail
Financial Coordinator

(405) 325-6029

ewjohnson@ou.edu 


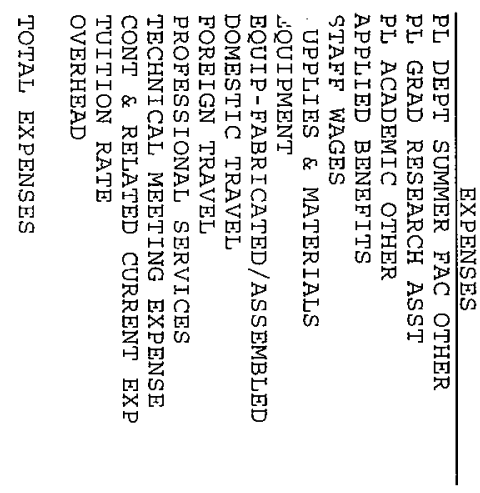

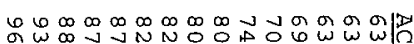
ONOUNANHNOOOOG
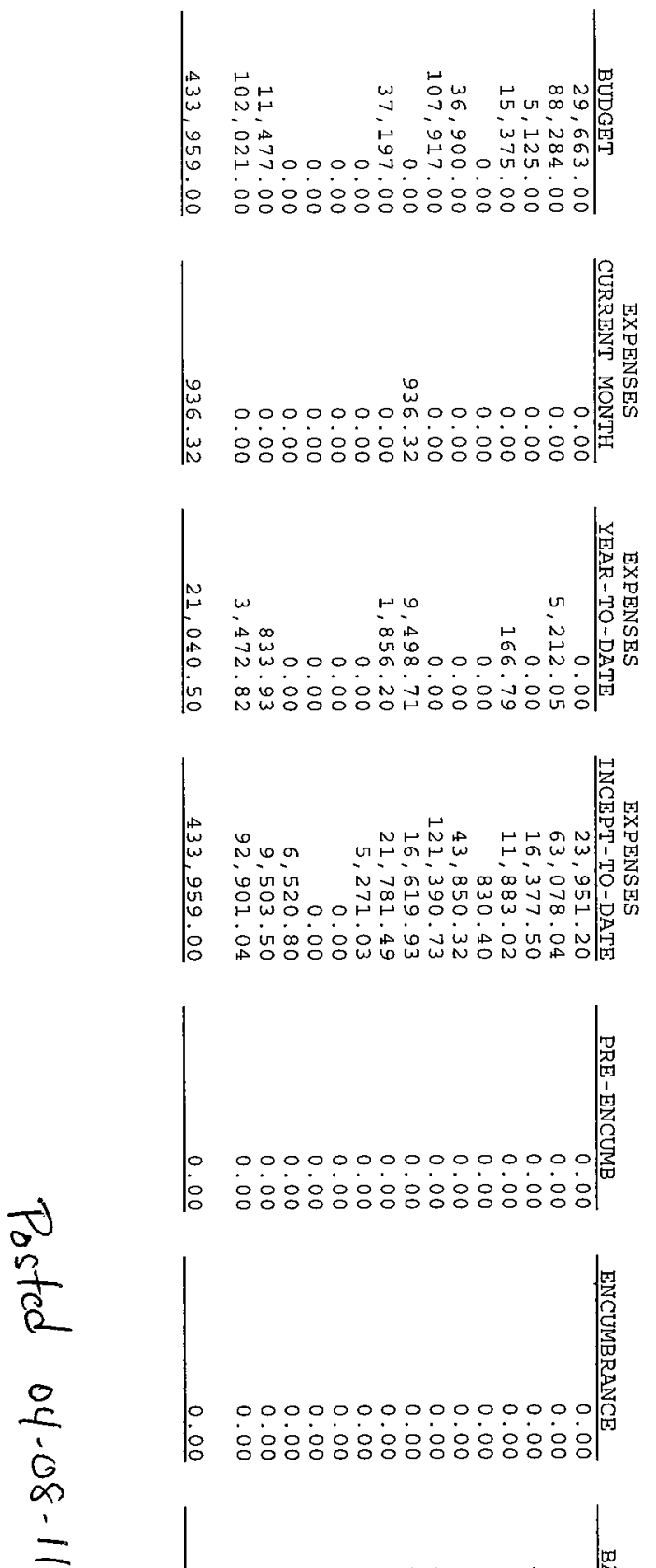

$\pi$

v
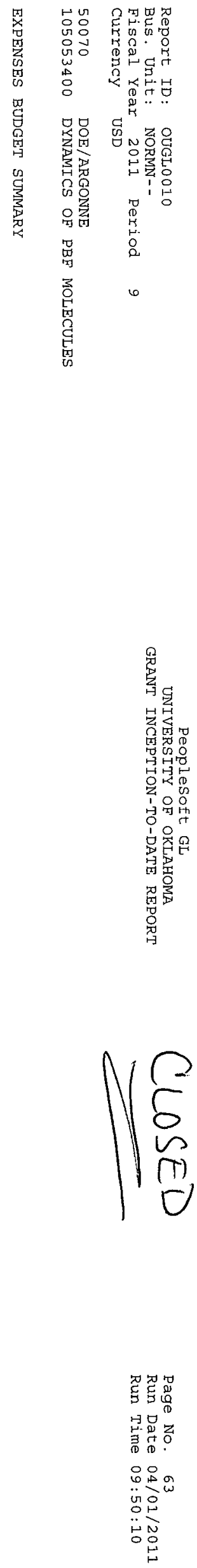


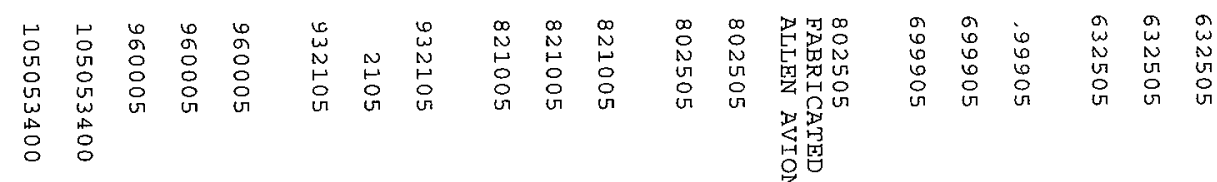

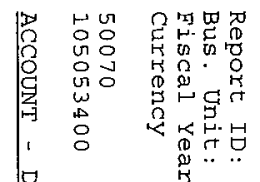

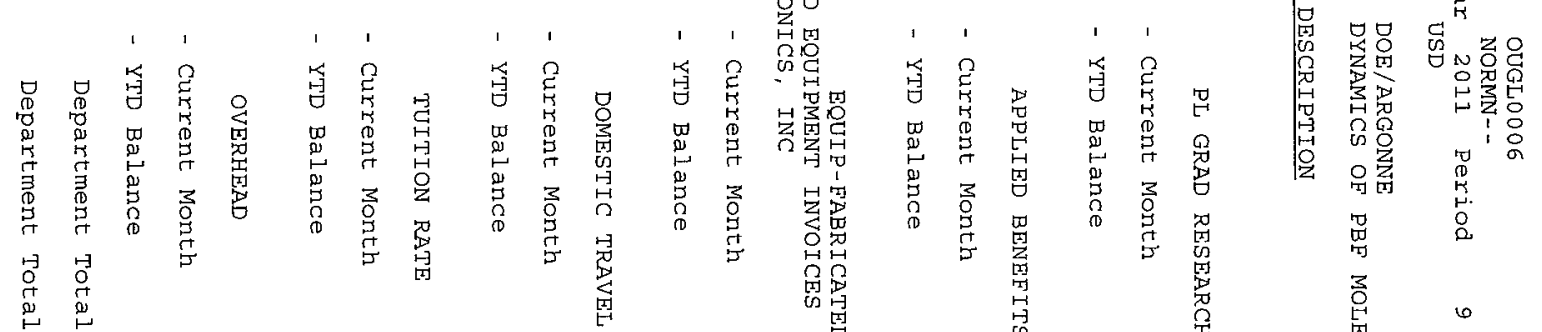

1

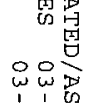

药

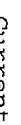

它占

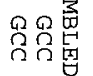

章

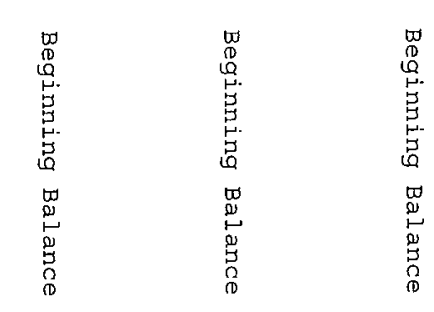

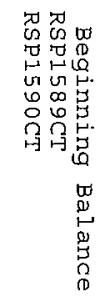

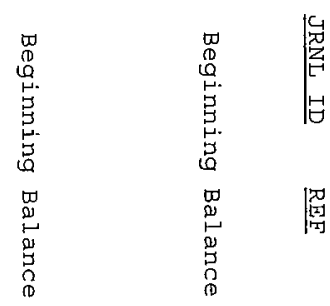

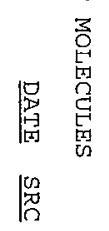

瓷

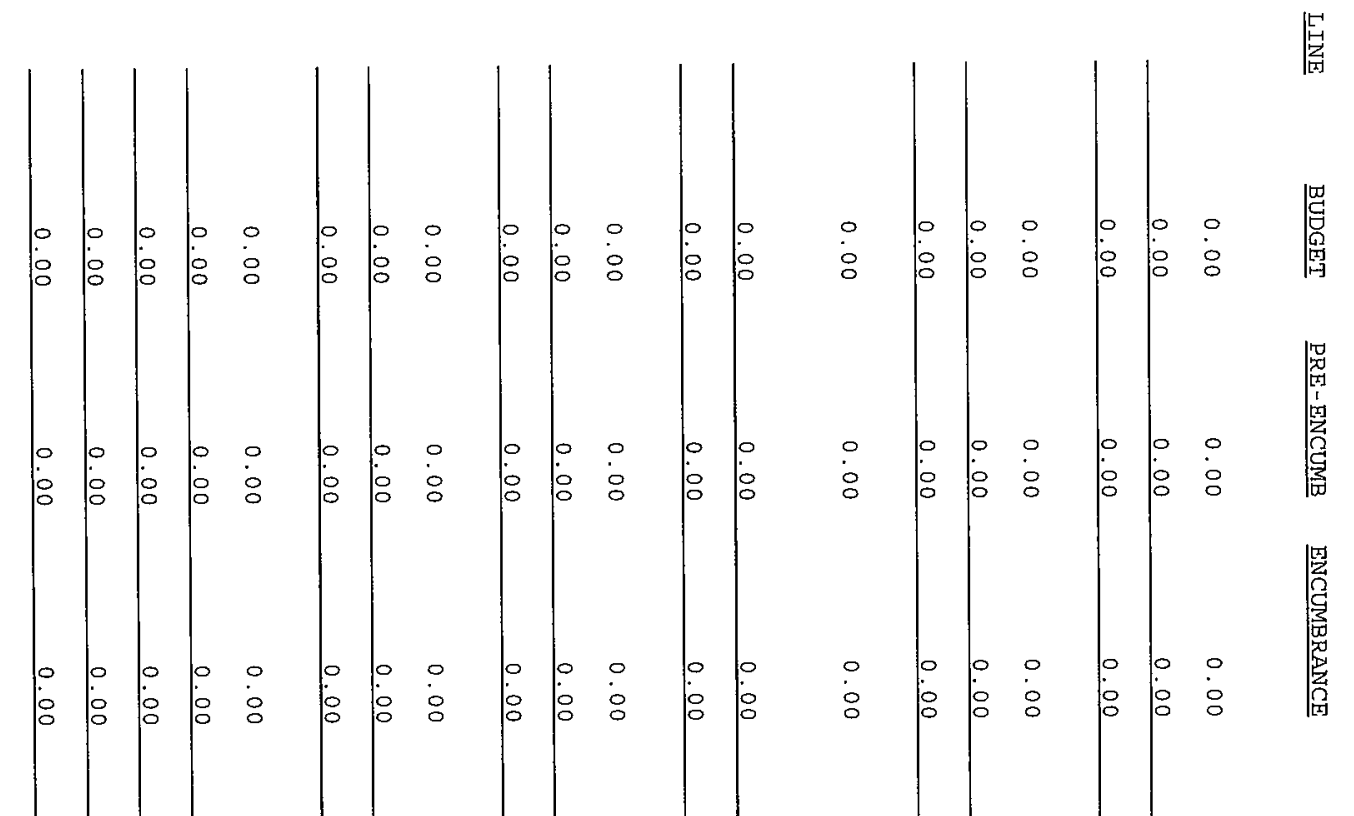

兽

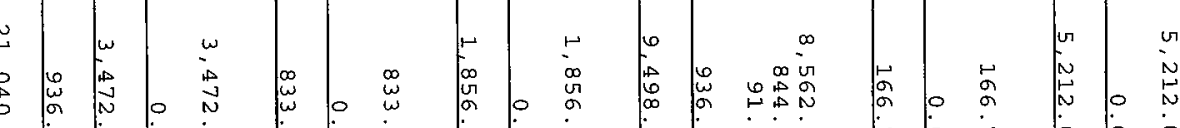

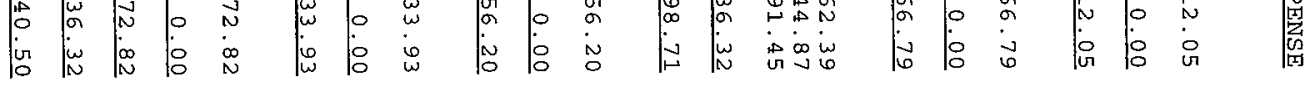

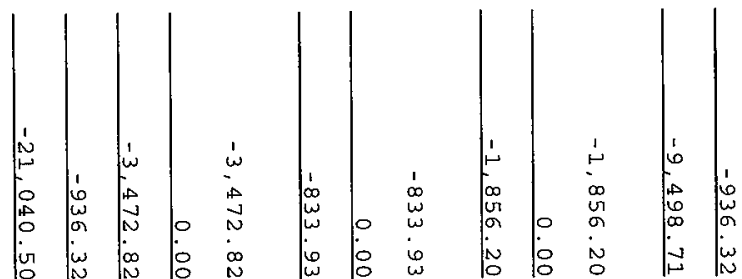

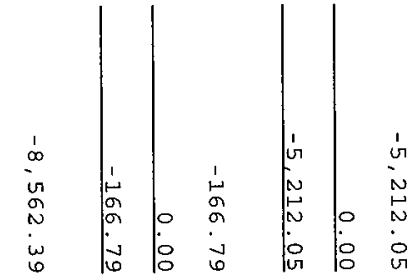

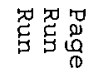

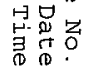

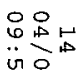

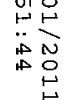




\section{BIBLIOGRAPHY}

[1] E. Purcell and N. Ramsey, Phys Rev 78, 807 (1950).

[2] Sakharov, JETP Lett. 5, 24 (1967).

[3] R. Arnowitt, B. Dutta, and Y. Santoso, Phys. Rev. D 64, 113010 (2001).

[4] F. Hoogeveen, Nuc. Phys. B341, 322 (1990).

[5] B. Regan, E. D. Commins, C. J. Schmidt, and D. DeMille, Phys. Rev. Lett. 88, 71805 (2002).

[6] M. Kozlov, V. Fomichev, Y. Y. Dmitriev, L. Labzovsky, and A. Titov, J. Phys. B: At. Mol. Opt. Phys. 20, 4939 (1987).

[7] N. Shafer-Ray, Phys. Rev. A 73, 34102 (2006).

[8] Longuet-Higgens, ed., Some Recent Developments in the Theory of Molecular Energy Levels, Advances in Spectroscopy II (Interscience Publishers, New York, 1961).

[9] I. Kopp and J. Hougen, Can. J. Phys. 45, 2581 (1967). 